\title{
Fully-Automatic Correction of the Erroneous Border Areas of an Aneurysm
}

\author{
J. Bruijns, F.J. Peters, R.P.M. Berretty, B. Barenbrug \\ Philips Research Eindhoven, The Netherlands \\ Email: Jan.Bruijns@philips.com
}

\begin{abstract}
Volume representations of blood vessels acquired by 3D rotational angiography are very suitable for diagnosing an aneurysm. We presented a fully-automatic aneurysm labelling method in a previous paper. In some cases, a portion of a "normal" vessel part connected to the aneurysm is incorrectly labelled as aneurysm. We developed a method to detect and correct these erroneous border areas. Application of this method gives better estimates for the aneurysm volumes.
\end{abstract}

\section{Problem}

Volume representations of blood vessels acquired by 3D rotational angiography show a clear distinction in gray values between tissue and vessel voxels [1]. These volume representations are very suitable for diagnosing an aneurysm. Physicians may treat an aneurysm by filling it with coils. Therefore, they need to know the volume of the aneurysm.

We developed a method for fully-automatic labelling of the aneurysm voxels after which the volume is computed by counting these voxels [2]. We use local distance thresholds to define a tight bounding surface around the aneurysm. This tight bounding surface should be located just outside the aneurysm where it borders to tissue. Elsewhere, it should intersect the "normal" vessel parts as close to the aneurysm as possible. The local distance thresholds are derived from border vessel voxels (i.e. vessel voxels connected to a tissue voxel). Since border vessel voxels are missing at an aneurysm neck, the tight bounding surface may bulge out into a "normal" vessel part. In such a case a portion of this vessel part is incorrectly labelled as aneurysm (Fig. 2.1, Fig. 2.3).

A possible solution to correct these erroneous labelled border areas of the aneurysm consists of first interactively creating a connection tube through such a border area, and next computing the neck outline. After the neck outline is computed, the aneurysm voxels in this border area are changed to "normal" vessel voxels [3]. The problem with this solution is that correcting all erroneous labelled border areas interactively may take a lot of time.

\section{Related work}

Bruijne et al. [4] use model-based interactive segmentation of abdominal aortic aneurysms from CTA data and multi-spectral MR images. After manual delin- 
eation of the aneurysm sac in the first slice, the method automatically detects the contour in subsequent slices.

Bescos et al. [5] described a method for the measurement of intracranial aneurysms from 3D rotational angiography using gradient edge detection. Using 13 aneurysm phantoms, they showed that their method gives more accurate volume measurements than gray value thresholds selected by a human operator. But, they use manual segmentation to separate the aneurysm from the "normal" vessels.

Wong et al. [6] described a method for the detection of vascular abnormalities given a topologically and morphologically correct vascular segmentation (i.e., with no holes and cavities). They first create a model of the "normal" vessels. The abnormal vascular structures are then determined as the complement of the approximated "normal" vessels. But, to create the initial tubes per vessel section a human operator has to select two centerline endpoints in $3 \mathrm{D}$ space for each section between two branch points. These centerline endpoints should be located outside the abnormal vascular structures.

\section{What is new}

To eliminate the time-consuming interaction, we have developed a method to automatically create as many of the required connection tubes as possible. For each created connection tube the neck outline is computed so that the erroneous aneurysm voxels in this border area are changed to "normal" vessel voxels.

We use the shape information extracted from the "normal" vessel parts to control this process. In our system, shape information extracted from a "normal" vessel part is stored in a tube object (tube for short). A tube consists of a series of consecutive probes [7]. A probe is a combination of a sphere, a plane through the center of the sphere and a number of shape parameters. After a tube is created by fully-automatic vessel tracing [8], the sphere centers are close to the central axis of the vessel, the planes are almost orthogonal to the vessel and each probe contains an ellipse representing the local cross-section of the probe's plane with the vessel surface. Note that the fully-automatic branch labelling method is extended to also create a node for each aneurysm neck.

\section{Method}

An erroneous labelled border area of the aneurysm is normally bounded by two necks. But not every neck pair defines an erroneously labelled border area. After all, there may exist neck pairs for which the connection tube travels through the inner area of the aneurysm (note that for faithful representation of the local vessel shape a connection tube does not connect the two necks but two remote cross-sections of the two "normal" vessel parts connected to these necks [3]). To prevent the inner area of the aneurysm from being changed, the necks of a border area neck pair must fulfill certain conditions. The most important ones are: 
Fig. 1. A checkpoint on a peripheral connection tube

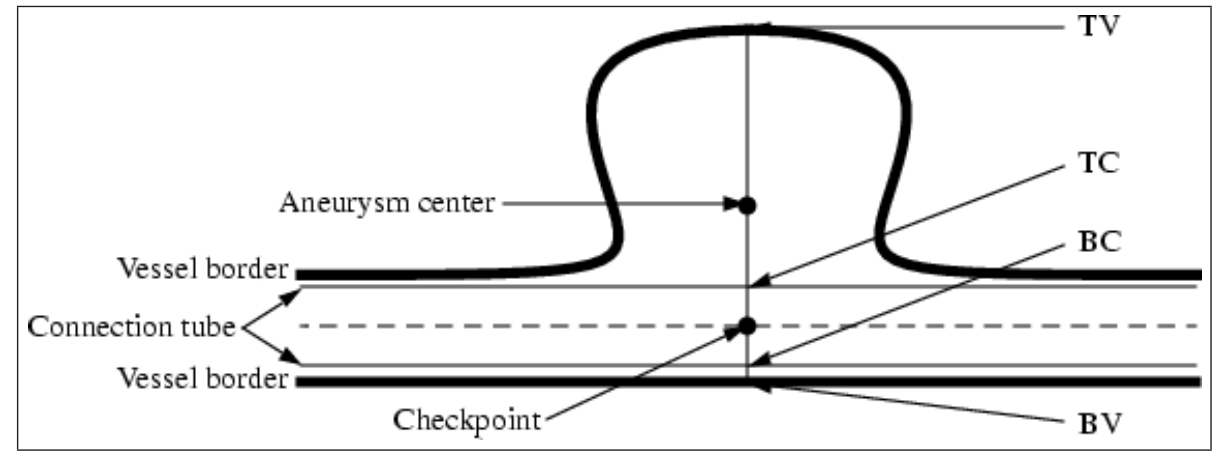

1. The vessel radii at the two neck nodes are about equal.

A neck pair is rejected if one radius is less than or equal to the half of the other radius. It is very unlikely that two such vessel parts represent an "normal" continuous vessel part.

2. The connection tube travels through the periphery of the aneurysm. We check the location of the connection tube at five checkpoints on the central curve between the two neck centers. A checkpoint is OK if one of the following two conditions is fulfilled along the line through this checkpoint and the center of the aneurysm (Fig. 1):

(a) The top of the aneurysm along this line $T V$ is located above the top of the connection tube $T C$ and the bottom of the connection tube $B C$ is located below the bottom of the vessel $B V$ (this case is not shown in Fig. 1).

(b) The distance below the connection tube ( $B C$ located above $B V$ as shown in Fig. 1) is less than the average neck radius $r$ and the distance above the connection tube is much greater than the distance below the connection tube: $\|B C-B V\|<r \wedge\|T V-T C\|>\|B C-B V\|+r$

The location of the vessel tube is OK if and only if all five checkpoints are OK.

\section{Remarks:}

1. If the aneurysm bulge does not block the "normal" vessel part completely, the erroneous labelled border area is defined by a single neck. So, not only all neck pairs but also each single neck is checked, applying similar conditions as used for each neck pair.

2. To detect as many erroneous labelled border areas as possible, the necks should be located as close to the aneurysm as possible (see [9]). Therefore, the necks have first been shifted as close to the real aneurysm as possible. 


\section{Results and discussion}

We have applied our method to 51 clinical volume datasets with a single aneurysm ( 16 of them with a resolution of $256 \times 256 \times 256$, the rest $128 \times 128 \times 128$ voxels), acquired with the 3D Integris system [10]. In 39 of these cases erroneous border areas were corrected. The total number of erroneous border areas corrected was 92 (an aneurysm can have more than one erroneous border area). No connection tube was generated through the inner area of an aneurysm.

The effect of the correction of the erroneous border areas can be perceived by comparing Fig. 2.1 with Fig. 2.2 and Fig. 2.3 with Fig. 2.4. These last two pictures reveal that our method is able to correct multiple connected erroneous border areas. Since the original vessel cross-sections are represented by the ellipses of the connection tube, tiny local bulges on the vessel surface result in a bumpy border between the aneurysm and the "normal" vessel section.

The mean number of aneurysm voxels changed is $12.5 \%$ of the number of aneurysm voxels, the maximum number of aneurysm voxels changed is $45.6 \%$ (a large relative correction may arise when the size of the real aneurysm is about the same as the size of the "normal" vessel part). Although some "normal" vessel voxels may be missed and some aneurysm voxels may be incorrectly changed, correction of the erroneous border areas gives better estimates for our clinical aneurysm volumes but a clinical validation of the accuracy of our aneurysm labelling method has yet to be done.

The average elapsed time for first shifting of the aneurysm necks as close to the real aneurysm as possible followed by fully-automatic correction of the erroneous border areas of an $128 \times 128 \times 128$ volume is 15.5 seconds on an SGI Octane $(300 \mathrm{MHz}$ MIPS R12000 + MIPS R12010 FPU) and 2.4 seconds on a Linux PC (2.8GHz Pentium 4). The average elapsed time for an 256x256x256 volume is 131.2 seconds on the SGI Octane and 16.6 seconds on the Linux PC. So, the elapsed time scales almost linearly with the number of voxels of the volume.

\section{References}

1. Kemkers R, de Beek JOp, Aerts H, et al. 3D-rotational angiography: First clinical application with use of a standard Philips C-arm system. In: Proc. CAR 98. Tokyo, Japan; 1998. 182-187.

2. Bruijns J. Local distance thresholds for enhanced aneurysm labelling. Procs BVM $2005 ; 148-152$.

3. Bruijns J, Peters FJ, Berretty RPM, et al. Computer-aided treatment planning of an aneurysm: The connection tube and the neck outline. In: Proc. VMV. Erlangen, Germany; 2005. 265-272.

4. de Bruijne M, van Ginneken B, Viergever MA, et al. Interactive segmentation of abdominal aortic aneurysms in CTA images. Med Image Anal 2004;8(2):127-138.

5. Bescos JO, Slob MJ, Slump CH, et al. Volume measurement of intracranial aneurysms from 3D rotational angiography: Improvement of accuracy by gradient edge detection. AJNR Am J Neuroradiol 2005;26(10):2569-2572. 
6. Wong WCK, Chung ACS. Augmented vessels for quantitative analysis of vascular abnormalities and endovascular treatment planning. IEEE Trans Med Imaging $2006 ; 25(6): 655-684$.

7. Bruijns J. Semi-automatic shape extraction from tube-like geometry. In: Proc. VMV. Saarbruecken, Germany; 2000. 347-355.

8. Bruijns J. Fully-automatic branch labelling of voxel vessel structures. In: Proc. VMV. Stuttgart, Germany; 2001. 341-350.

9. Bruijns J, Peters FJ, Berretty RPM, et al. Shifting of the aneurysm necks for enhanced aneurysm labelling. Procs BVM 2006; 141-145.

10. Philips-Medical-Systems-Nederland. INTEGRIS 3D-RA. Instructions for use. Release 2.2. Philips Medical Systems Nederland. Best, The Netherlands; 2001.

\section{Pictures}

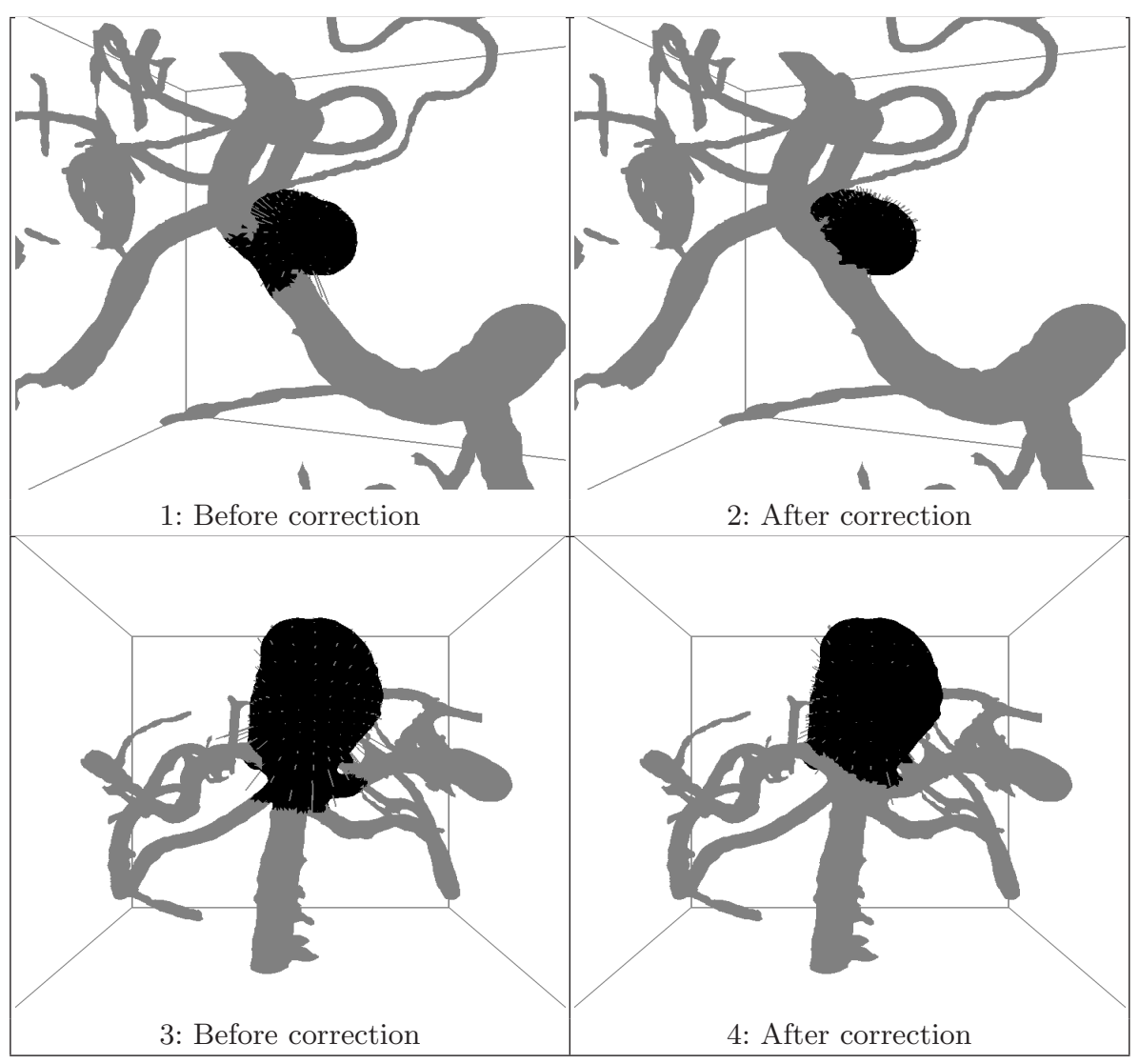

Fig. 2. Two aneurysm cases 